\title{
AN EFFICIENT PRECONDITIONING STRATEGY FOR SCHUR COMPLEMENTS ARISING FROM BIPHASIC MODELS
}

\author{
Marco Favino \\ Institute of Computational Science \\ University of Lugano \\ via Buffi, 13 \\ Lugano, Canton Ticino, Switzerland \\ email: favinom@usi.ch
}

\author{
Rolf Krause and Johannes Steiner \\ Institute of Computational Science \\ University of Lugano \\ via Buffi, 13 \\ Lugano, Canton Ticino, Switzerland \\ email: \{rolf.krause,johannes.steiner\}@usi.ch
}

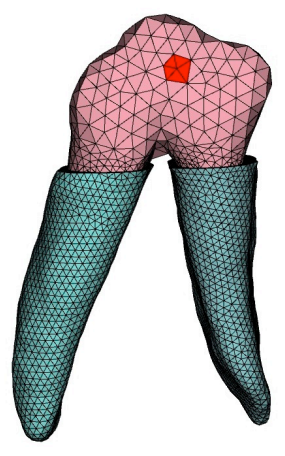

(a) Labial side

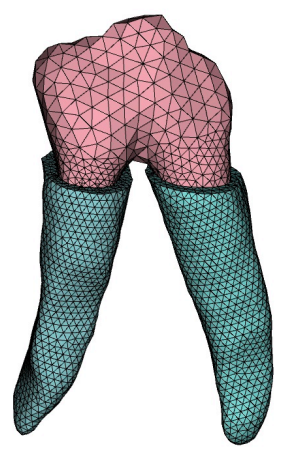

(b) Buccal side

\section{KEY WORDS}

biphasic model, periodontal ligament, finite element method, Schur complement, elastic-biphasic coupling.

\section{Introduction}

The Periodontal Ligament (PDL) is a thin layer of dense soft connective tissue located between tooth root and alveolar (jaw) bone, see Figure 1. Its bio-mechanical functions are diverse [2]. Firstly, it supports the teeth and keeps them in position. Secondly, it allows for attenuating the high and fast loading of the teeth during chewing. Thirdly, the PDL transmits the long-term loading to the alveolar bone thus enabling bone-remodeling processes.

Understanding the behavior and the mechanical properties of the PDL is of paramount interest not only from a medical point of view, e.g. for investigating diseases of the PDL as periodontitis, but also in the context of biomechanical applications as, e.g., estimating the maximal loading applicable with dental braces. Since in-vivo biomechanical experiments with humans are possible only within strict limits, mathematical modeling and numerical simulation are important tools for creating a deeper understanding of the mechanical nature of the PDL.

As a matter of fact, the specific material behavior of the PDL are based on its biphasic structure. Internally the PDL consists of a solid part, which is formed by a fibrous tissue, and a fluid phase. While the fibrous network provides structural support, the fluid phase adds hydrodynamic damping, thus allowing for different reactions of the PDL to short-term and long-term loading.

This combination turns the mathematical modeling and the numerical simulation of the PDL into a demanding task, since not only the material properties of the fibruous tissue have to be covered but also its interaction with the fluid phase. In terms of unknowns of the resulting system of partial differential equations, i.e. the displacements of the fibrous tissue and the pressure of the mixture, this interaction gives rise to a non-linear coupling term, since the permeability depends non-linearly on the displacements.

From a numerical point of view, the most convenient way to deal with this non-linearity is to treat it explicitely within the time integration scheme. Unfortunately this procedure is not always stable and suitable linearization strategies has to be employed [7]. Then, discretizing the linearized equation with finite elements at each time step, a saddle point problem arises. For realistic applications, however, due to the high number of degrees of freedom, direct solution strategies are inapplicable and one has to resort to iterative solution schemes which exploit the structure of the system. Several preconditioning strategies for saddle point problems can be found in the literature e.g. [1]. In the context of multigrid methods, transforming 
smoothers or point block-smoothers can be found as well as approaches based on Uzawa's method with multigrid as "inner" solver [4].

Our approach follows the latter ideas and is based on solving the Schur complement of the biphasic system. In order to take into account the pressure of the mixture, in contrast to standard approaches we do not only use mass matrix as a preconditioner for the Schur complement, but the sum of a scaledmass matrix and the discretized diffusion operator related to the pressure. As can be seen from our numerical experiments, our new preconditioning strategy behaves optimally with respect to the mesh size.

In this contribution, we start from the derivation of a suitable biphasic model for the PDL. For two model problems we show that our preconditioning approach gives rise to mesh independent convergence rates. We conclude with a "real world" application of the derives techniques within the context of dental biomechanics.

Here we want to point out that the presented preconditioner can be employed not only for the biphasic system but also for other physical problems with the same saddle point structure, e.g. the Brezzi-Pitkärenta stabilization of the Stokes problem [5].

\section{Modeling of the PDL}

In this section we will briefly introduce the model describing the tooth-PDL system. For a detailed description of porous elasticity see [3], for the employed model [6] and reference therein while for the dental applications see our work [7]. Therein we performed some simulations with realistic elastic parameters obtained by measurements on porcine tooth and PDL. These simulations allowed us also to detect some feasible values for the unknown permeability parameters.

\subsection{Kinematics}

According to theory of porous media that we employ to model the periodontal ligament (PDL), the fibrous network and the fluid phase within the PDL are modeled by assuming that the solid and the fluid phase coexist in a homogenized way throughout the PDL. We assume that every point $\boldsymbol{p}$ of the reference configuration $\Omega_{B}$ of the PDL is occupied by a "material point" of both phases $\alpha$, where $\alpha$ can be either $S$, the solid phase, or $F$, the fluid phase, respectively. Each point $\boldsymbol{p}$ is therefore moved according to two different motions $\boldsymbol{f}_{\alpha}(\boldsymbol{p}, t), \alpha \in S, F$, see Figure 2. Every spatial point $\boldsymbol{x}$ at time $t$ of the current deformed configuration $\Omega_{B}^{t}$ is therefore occupied by two different fields with

$$
\boldsymbol{f}_{S}\left(\Omega_{B}, t\right)=\boldsymbol{f}_{F}\left(\Omega_{B}, t\right) \text { for all } t \geq 0 .
$$

As a consequence, at every spatial point two different velocity fields coexist which will be denoted by $\boldsymbol{v}_{\alpha}$ in spatial coordinates.

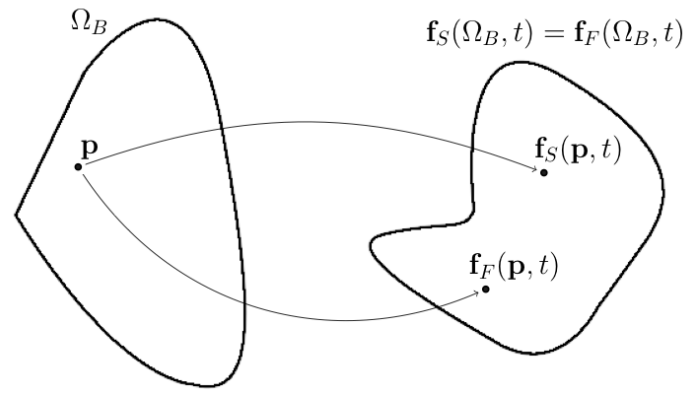

Figure 2. At each material point the two phases coexist and result in two different deformations $\boldsymbol{f}_{S}$ and $\boldsymbol{f}_{F}$.

The local structure of the fluid-solid mixture is assumed to be governed by the volume fractions $n_{\alpha}=$ $n_{\alpha}(\boldsymbol{x}, t)$ that describe the ratio of the fluid and solid phase at each spatial point. To ensure that there is no empty space between the phases we employ the usual saturation condition

$$
n_{S}+n_{F}=1
$$

that has to be fulfilled. The evolution of the solid volume fraction $n_{S}$ can be computed starting from the fraction $n_{S}^{0}(\boldsymbol{p}, t)$ in the reference configuration as

$$
n_{S}=n_{S}^{0} \operatorname{det}\left(\nabla \boldsymbol{f}_{S}\right)^{-1} .
$$

Exploiting twice the saturation condition, the evolution of the fluid fraction can be computed as

$$
n_{F}=n_{F}^{0}+n_{S}^{0} \operatorname{det}\left(\nabla \boldsymbol{f}_{S}\right)^{-1},
$$

where $n_{0}^{F}$ denotes the initial volume fractions of the fluid phase. Thus, the density at each point $\boldsymbol{x}$ is given by

$$
\rho=n_{S} \rho_{S}^{P}+n_{F} \rho_{F}^{P}
$$

where $\rho_{\alpha}^{P}$ denotes the density of each phase $\alpha$, which is constant in the incompressible case. As is standard in continuum mechanics and fluid mechanics, respectively, the motion of the solid part is described in terms of the displacements $\boldsymbol{u}_{S}=\boldsymbol{f}_{S}(\boldsymbol{p}, t)-\boldsymbol{p}$ while the velocity of the fluid is given in term of the seepage velocity $\boldsymbol{w}_{F}=\boldsymbol{v}_{F}-\boldsymbol{v}_{S}$.

\subsection{Balance Equations}

The balance equations have to be solved for both of the phases $S$ and $F$. Starting from the conservation of mass

$$
\rho_{\alpha}^{\prime}(\boldsymbol{x}, t)+\operatorname{div}\left(\rho_{\alpha}(\boldsymbol{x}, t) \boldsymbol{v}_{\alpha}(\boldsymbol{x}, t)\right)=0
$$

and exploiting the incompressibility condition we arrive at

$$
n_{\alpha}^{\prime}(\boldsymbol{x}, t)+\operatorname{div}\left(n_{\alpha}(\boldsymbol{x}, t) \boldsymbol{v}_{\alpha}(\boldsymbol{x}, t)\right)=0 .
$$

Summing up the respective equations (2) for both of the two phases, we obtain

$$
\left(n_{S}+n_{F}\right)^{\prime}+\operatorname{div}\left(n_{S} \boldsymbol{v}_{S}+n_{F} \boldsymbol{v}_{F}\right)=0 .
$$


Now, employing the saturation condition (1) and the definition of $\boldsymbol{w}_{F}$, we get

$$
\operatorname{div}\left(\boldsymbol{v}_{S}(\boldsymbol{x}, t)+n_{F}(\boldsymbol{x}, t) \boldsymbol{w}_{F}(\boldsymbol{x}, t)\right)=0 .
$$

For each constituent, neglecting inertia and volume forces, the balance of momentum is written as

$$
-\operatorname{div} \boldsymbol{T}_{\alpha}+\boldsymbol{\Pi}_{\alpha}=0,
$$

where $\Pi_{\alpha}$ denotes the momentum production term which fulfills $\boldsymbol{\Pi}_{S}+\boldsymbol{\Pi}_{F}=0$ and $\boldsymbol{T}_{\alpha}$ is the Cauchy stress tensor. Due to the assumption of incompressibility, the pressure is introduced and the Cauchy tensor can be written as

$$
\boldsymbol{T}_{\alpha}^{E}-n_{\alpha} p \boldsymbol{I}
$$

where $\boldsymbol{T}_{\alpha}^{E}$ is the elastic stress tensor that can be neglected for the fluid phase.

\subsection{Coupled Model for Tooth and Periodontal Liga- ment}

In order to describe the complete PDL-tooth system, the biphasic model has to be completed by elastic model for the tooth. We denote by $\Omega_{E} \subset \mathbb{R}^{3}$ the reference domain of the elastic material and associate all related quantities with the same subscript. Because of the small strain and small displacement occurring, a geometrically linear elastic model can be employed

$$
-\nabla \cdot \boldsymbol{T}_{E}^{E}=0 .
$$

The vector $\boldsymbol{u}_{E}$ denotes the displacement of the elastic domain $\Omega_{E}$ and the following compatibility conditions on the interface $\Gamma^{I}=\overline{\Omega^{\text {Tooth }}} \cap \overline{\Omega^{\text {PDL }}}$ between the tooth and the PDL have to be fulfilled:

$$
\begin{aligned}
\boldsymbol{u}_{S} & =\boldsymbol{u}_{E} & & \text { on } \Gamma^{I} \\
\left(\boldsymbol{T}_{S}+\boldsymbol{T}_{F}\right) \cdot \boldsymbol{\nu}_{\mathrm{B}} & =-\boldsymbol{T}_{T}^{E} \cdot \boldsymbol{\nu}_{E} & & \text { on } \Gamma^{I}
\end{aligned}
$$

Here, $\nu_{\mathrm{B}}$ and $\boldsymbol{\nu}_{E}$ denote the outward normals to $\Omega_{B}$ and to $\Omega_{E}$, respectively.

\subsection{Constitutive Equations}

In order to describe the elastic behavior of the tooth and the solid phase of the PDL, a geometric linear elastic model is used, i.e. the strain is measured by means of the linearized strain tensor

$$
\boldsymbol{\epsilon}_{\beta}=\boldsymbol{\epsilon}\left(\boldsymbol{u}_{\beta}\right)=\frac{1}{2}\left(\nabla \boldsymbol{u}_{\beta}+\left(\nabla \boldsymbol{u}_{\beta}\right)^{T}\right) .
$$

with $\beta \in\{S, E\}$. This simplification, which is reasonable within the limits of our model, allows us to write the elastic part of the Cauchy tensor as

$$
\boldsymbol{T}_{\beta}^{E}=2 \mu_{\beta} \boldsymbol{\epsilon}_{\beta}+\lambda_{\beta} \operatorname{tr}\left(\boldsymbol{\epsilon}_{\beta}\right) \boldsymbol{I}
$$

As a next step, we need a closure relation that allows us to connect the seepage velocity to the pressure in the mixture. To this end the well-known Darcy law is obtained by the balance of momentum of the fluid phase and can be written

$$
n_{F} \boldsymbol{w}_{F}=-\zeta\left(n_{f}\right) \operatorname{grad} p
$$

The parameter $\zeta$ describes the permeability of the solid phase. To model this term different suggestions can be found in the literature. The model we are using is the one proposed in [8] and reads

$$
\zeta\left(n_{F}\right)=\frac{k_{F}\left(n_{F}\right)}{\gamma_{F}^{R}} .
$$

The numerator describes the evolution of permeability with zero displacement $k_{0}^{F}$ with a power law

$$
k_{F}\left(n_{F}\right)=k_{F}^{0}\left(\frac{n_{F}}{n_{F}^{0}}\right)^{\kappa}
$$

while the denominator is the effective pressure of the fluid.

Summarizing, we now can state the system of partial differential equations describing the linear biphasic model without considering the volume forces as follows:

$$
\begin{aligned}
-\operatorname{div}\left(\mu_{S} \boldsymbol{\epsilon}_{S}+\lambda_{S} \operatorname{tr}\left(\boldsymbol{\epsilon}_{S}\right) \boldsymbol{I}-p \boldsymbol{I}\right) & =\mathbf{0} \\
\operatorname{div}\left(\boldsymbol{v}_{S}-\zeta\left(n_{F}\right) \operatorname{grad} p\right) & =0
\end{aligned}
$$

Please note that although in (3) we are in the geometric and material linear case, the above system is non-linear since the permeability parameter is non-linear function of the solid displacement $\boldsymbol{u}_{S}$.

The parameters employed for the simulation of the tooth-PDL model are reported in table 1. The mechanical parameters have been obtained by measurements on porcine tooth while the parameters describing the permeability model are the ones in the ranges presented in [7] that was giving results in good agreement with the physical experiments.

\begin{tabular}{c|c|c}
\hline Parameter & value & units \\
\hline \hline$\mu_{S}$ & $0.08 \times 10^{6}$ & $\mathrm{~N} \mathrm{~m}^{-2}$ \\
\hline$\lambda_{S}$ & $0.11 \times 10^{6}$ & $\mathrm{~N} \mathrm{~m}^{-2}$ \\
\hline$\mu_{S}$ & $8.00 \times 10^{9}$ & $\mathrm{~N} \mathrm{~m}^{-2}$ \\
\hline$\lambda_{S}$ & $11.00 \times 10^{9}$ & $\mathrm{~N} \mathrm{~m}^{-2}$ \\
\hline$\rho_{F}^{R}$ & 1000 & $\mathrm{~kg} \mathrm{~m}^{-3}$ \\
\hline$k_{F}^{0}$ & $10^{-6} \div 10^{-8}$ & $\mathrm{~m} \mathrm{~s}^{-1}$ \\
\hline$\kappa$ & $30 \div 40$ & $\#$ \\
\hline$n_{0}^{S}$ & 0.6 & $\#$ \\
\hline$n_{0}^{F}$ & 0.4 & $\#$ \\
\hline
\end{tabular}

Table 1. Physical parameters used in the numerical experiments 


\section{Discretization in Space and Time}

Here, we provide a brief description of the discretization in space and time used for our numerical experiments. For the sake of clarity, in this presentation we will consider $\lambda=0$ and $\mu$ constant but the strategy can be easily extended to more general cases. A simple implicit first order discretization in time by means of the backward Euler method gives rise at each time step $j$ to the following non-linear system of partial differential equations:

$$
\begin{aligned}
-\operatorname{div}\left(2 \mu \boldsymbol{\epsilon}\left(\boldsymbol{u}_{S}^{j}\right)-p^{j} \boldsymbol{I}\right) & =0 \\
\operatorname{div}\left(\boldsymbol{u}_{S}^{j}-\Delta t \zeta\left(n_{f}^{j}\right) \operatorname{grad} p^{j}\right) & =\operatorname{div} \boldsymbol{u}_{S}^{j-1}
\end{aligned}
$$

As has been observed in [7] a purely explicit treatment of the non-linearity in the permeability $\zeta$ leads to a non-stable solution. In order to overcome this difficulty, we therefore employ a predictor-corrector scheme, see again [9]. As a matter of fact, numerical evidence shows that even for simulations on complicated geometries with realistic material parameters one predictor and one corrector step are sufficient for ensuring stability.

For the discretization in space, we employ low order finite elements for the displacements as well as for the pressure. To this end, the computational domain is approximated by an unstructured mesh with tetrahedral elements. On $\Omega_{B}$, where we solve the biphasic model, at each mesh node we have three degrees of freedom for the displacements and one for the pressure. This discretization of the biphasic model gives rise to the following system with saddle point structure:

$$
\left(\begin{array}{cc}
\mu \boldsymbol{A} & -\boldsymbol{B}^{T} \\
\boldsymbol{B} & \Delta t k_{F}^{0} \boldsymbol{C}^{\beta}
\end{array}\right)\left(\begin{array}{l}
\boldsymbol{x} \\
\boldsymbol{p}
\end{array}\right)=\left(\begin{array}{l}
\boldsymbol{f} \\
\boldsymbol{g}
\end{array}\right)
$$

At every time step $j$, the value of $\beta$ is $j-1$ for the predictor step and $j-1 / 2$ for the corrector step. For details on the linear system resulting from the discretization of the biphasic-elastic coupled model see [7].

\section{Preconditioning Strategy}

Due to the huge number of degrees of freedom for realistic PDL-tooth geometries, iterative solvers in combination with efficient preconditioning strategies have to be employed. Multigrid methods, for example, are well known to be preconditioners and solvers providing optimal complexity for elliptic problems. For indefinite systems, however, the direct application of multigrid solvers is still a challenge since the choice of appropriate smoothers is far from trivial, since classical iterative solvers as, e.g. Jacobi, GaussSeidel, SOR, are designed for positive definite matrices.

In order to provide an efficient preconditioning strategy, we employ the Schur complement of (4)

$$
\boldsymbol{S}=\frac{1}{\mu} \boldsymbol{B} \boldsymbol{A}^{-1} \boldsymbol{B}^{T}+\Delta t k_{F}^{0} \boldsymbol{C} .
$$

Assuming a constant shear modulus in space, we can characterize the material dependencies in the problem by means of the single dimensionless permeability parameter $k=$ $\Delta t k_{F}^{0} \mu$. The matrix $\boldsymbol{S}$ is symmetric and positive definite by construction. Thus, if a good preconditioner for the elasticity stiffness matrix $\boldsymbol{A}$ is available, the preconditioned conjugate gradient method will provide an efficient solution method. The spectral behavior of $\boldsymbol{B} \boldsymbol{A}^{-1} \boldsymbol{B}^{T}$ is well known in case of constant coefficients and it can be seen to be equivalent to that of the corresponding mass matrix. For the biphasic model considered here, also the additional matrix $C$ has to be taken into account. Since $C$ is an additional diffusion matrix, we choose as preconditioner the sum of the pressure mass matrix $\boldsymbol{M}$ (scaled by $1 / \mu$ ) and $C$ (scaled by $k_{F}^{0} \Delta t$ ). The resulting matrix is symmetric positive definite again and multigrid method can be applied.

In the next section we will perform several numerical test and we will investigate the performance of our approach with respect to the discretization size $h$ for the simulation of the biphasic model. We will also perform some numerical test with non-constant $\mu$. In this case $\mu$ cannot be eliminated in the Schur complement. We circumvent this difficulty by employing as "mass" preconditioner the discretization of weighted scalar product

$$
\int_{\Omega_{B}} \frac{1}{\mu} u v \mathrm{~d} x
$$

Let us finally remark that using a symmetric positive definite matrix for preconditioning as described above together with a multigrid method as solution method can render the solution of the biphasic model optimal.

\section{Numerical Results}

In this paragraph, we investigate our preconditioning strategy along a model problem with realistic parameters. We moreover apply the derived model to a real-world problem.

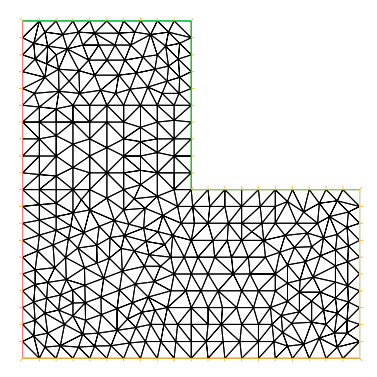

Figure 3. L-shape domain.

The first example to be considered is a model problem in 2D. As computational domain we choose an L-shaped domain (see Figure 3). We use the biphasic material model derived in Section 2. The material parameter $k$ varies in the range from $10^{-1}$ to $10^{-5}$. This range is designed to cover 


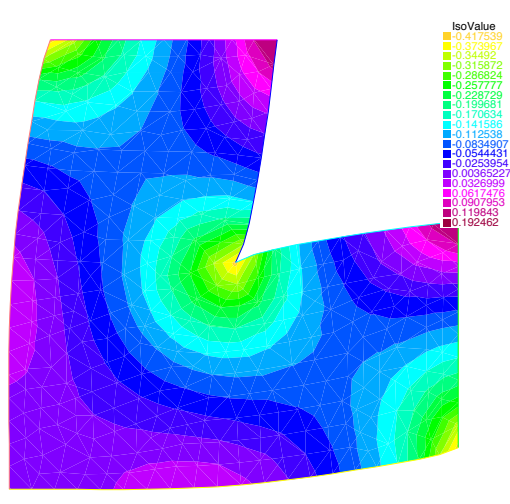

Figure 4. Pressure on the deformed domain.

\begin{tabular}{|c|c|c|}
\hline $\mathrm{h}$ & precon. & mass precon. \\
\hline \hline 0.2 & 3 & 41 \\
\hline 0.1 & 3 & 81 \\
\hline 0.05 & 3 & 169 \\
\hline 0.025 & 3 & 346 \\
\hline 0.0125 & 3 & 732 \\
\hline 0.00625 & 3 & $1,000^{*}$ \\
\hline
\end{tabular}

Table 2. L-Shaped domain: Number of iteration with $k=$ 0.1

realistic material parameters occurring in dental biomechanics. For this L-shaped domain we use an unstructured and regular triangular mesh with mesh size $h>0$. An example of the computed displacements and pressure is reported in Figure 4. The second example is given by a unit square in $2 \mathrm{D}$. This domain is split up into two parts of equal size on which different shear moduli are prescribed. In this case we use regular mesh with mesh size $h$.

Along this examples, we investigate our preconditioning strategy by measuring the number of iterations for different solution strategies. More precisely, we compare a conjugate gradient $(\mathrm{cg})$ method on the Schur-complement with a preconditioned cg-method where we have used $M$ and $\boldsymbol{M}+k \boldsymbol{C}$ as a preconditioner, respectively. In all cases we stop the iteration process if the relative error in the residual of the linear system is below $10^{-6}$. As we will see, the number of iterations can be reduced significantly by the application of our preconditioning strategy which then will allow for the application of the biphasic model to a real world problem.

In Tables 2, 3 and 4 the number of iterations for decreasing mesh size $h$ and three different permeability parameters for the L-Shaped domain are given. Uniform refinement is applied.

In Tables 5 and 6 the number of iterations for decreasing mesh size $h$ and two different permeability parameters for the example with jumping coefficients are given. Again, uniform refinement is applied. As can be seen, the number of iterations are remains constant by the application of our

\begin{tabular}{|c|c|c|c|}
\hline $\mathrm{h}$ & precon. & mass precon. & unprecon. \\
\hline \hline 0.2 & 8 & 10 & 16 \\
\hline 0.1 & 8 & 18 & 22 \\
\hline 0.05 & 7 & 40 & 40 \\
\hline 0.025 & 7 & 85 & 80 \\
\hline 0.0125 & 7 & 180 & 157 \\
\hline 0.00625 & 7 & 375 & 322 \\
\hline
\end{tabular}

Table 3. L-Shaped domain: Number of iteration with $k=$ 0.001

\begin{tabular}{|c|c|c|c|}
\hline $\mathrm{h}$ & precon. & mass precon. & unprecon. \\
\hline \hline 0.2 & 34 & 53 & 62 \\
\hline 0.1 & 23 & 40 & 51 \\
\hline 0.05 & 14 & 22 & 34 \\
\hline 0.025 & 10 & 11 & 20 \\
\hline 0.0125 & 9 & 18 & 20 \\
\hline 0.00625 & 9 & 39 & 38 \\
\hline
\end{tabular}

Table 4. L-Shaped domain: Number of iteration with $k=$ 0.00001

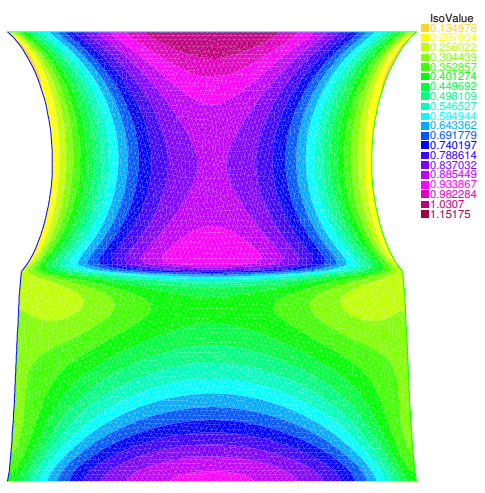

Figure 5. Jumping material parameters: traction of a square with two different shear moduli.

strategy. It can furthermore be seen that the number of iterations is independent of the mesh size, but depends on the dimensionless permeability parameter $k$.

In Figure 6 a simulation on a complete PDL-tooth system is given. In the tooth domain the von Mises stress are plotted: the area with higher stress on the frontal (labial) surface is the contact area with the loading device simulated as a boundary Dirichlet boundary condition while in the PDL domain the pressure in the PDL is given. For a detailed validation of a coupled biphasic-elastic model see [7].

The aim of our real-world numerical examples is the reproduction of in-vitro measurements [7]. In these experiments, time-dependent displacements are applied to the tooth and concurrently the force response on the tooth is measured at the position of the bolt. 


\begin{tabular}{|c|c|c|c|}
\hline $\mathrm{h}$ & $\mu=10$ & $\mu=100$ & $\mu=1000$ \\
\hline \hline 0.2 & 27 & 33 & 33 \\
\hline 0.1 & 20 & 19 & 18 \\
\hline 0.05 & 13 & 13 & 12 \\
\hline 0.025 & 11 & 11 & 11 \\
\hline 0.0125 & 11 & 11 & 11 \\
\hline 0.00625 & 11 & 11 & 11 \\
\hline
\end{tabular}

Table 5. Number of iterations, for two different shear moduli $\mu_{1}$ and $\mu_{2}$. The value of $k$ is $10^{-4}$ and $\mu=\mu_{2} / \mu_{1}$.

\begin{tabular}{|c|c|c|c|c|}
\hline $\mathrm{h}$ & $\mu=1$ & $\mu=2$ & $\mu=10$ & $\mu=100$ \\
\hline \hline 0.2 & 18 & 27 & 27 & 33 \\
\hline 0.1 & 19 & 20 & 20 & 19 \\
\hline 0.05 & 12 & 12 & 13 & 13 \\
\hline 0.025 & 10 & 11 & 11 & 11 \\
\hline 0.0125 & 10 & 11 & 11 & 11 \\
\hline 0.00625 & 11 & 11 & 11 & 11 \\
\hline
\end{tabular}

Table 6. Number of iterations, for two different shear moduli $\mu_{1}$ and $\mu_{2}$. The value of $k$ is $10^{-3}$ and $\mu=\mu_{2} / \mu_{1}$.

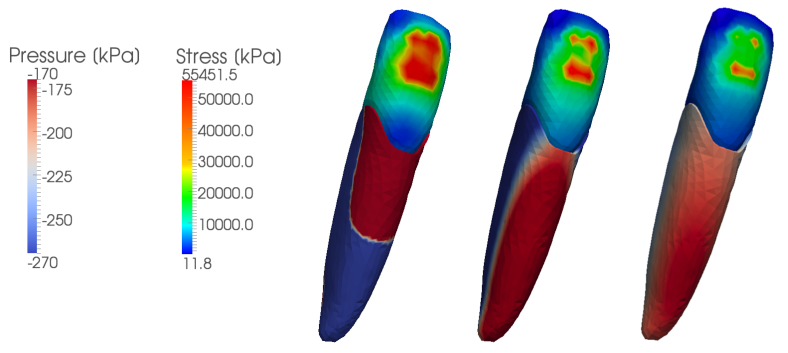

Figure 6. The evolution of von Mises stress in the tooth and the pressure in the PDL in the simulation of a relaxation test on a single-rooted tooth.

In these numerical simulations, the contact surface between the indenter and the tooth is modeled by a time-dependent Dirichlet boundary condition reaching a maximum displacement of $0.10 \mathrm{~mm}$.

The contact between the PDL and the surrounding jaw bone is modeled by means of homogeneous Dirichlet boundary conditions. In this study, we furthermore neglect the flow of the interstitial fluid through the surface between the PDL and the bone.

\section{Conclusions}

In this work we have considered the mathematical modeling of the periodontal ligament and its numerical simulation. We have investigated a preconditioning strategy for the Schur complement of the saddle point problem arising after discretization in space and time. Our strategy showed to be optimal in different numerical test, thus allowing for the simulation of "real world" problems from dental medicine. Future extensions of our work will include the improvement of the material model and the development of corresponding fast solution methods.

\section{References}

[1] Michele Benzi and Andrew J. Wathen. Model Order Reduction: Theory, Research Aspects and Applications, chapter Some Preconditioning Techniques for Saddle Point Problems. Springer Verlag, 2008.

[2] B. K. B. Berkovitz, G. R. Holland, and B. J. Moxham. A Colour Atlas and Textbook of Oral Anatomy. Histology and Embryology. Wolfe Medical Publications, London, 2nd edition, 1992.

[3] R. Bowen. Porous Elasticity: Lectures on the elasticity of porous materials as an application of the theory of mixtures. Available electronically from http://hdl.handle.net/1969.1/91297, 2010.

[4] D. Braess and R. Sarazin. An efficient smoother for the Stokes problem. Applied Numerical Mathematics, 23(1):3 - 19, 1997. Multilevel Methods.

[5] F. Brezzi and J.Pitkäranta. On the stabilization of finite element approximations of the Stokes equations. In W. Hackbusch, editor, Efficient Solutions of Elliptic Systems, pages 11-19. Vieweg, 1984.

[6] W. Ehlers and B. Markert. A linear viscoelastic biphasic model for soft tissues based on the Theory of Porous Media. ASME Journal of Biomechanical Engineering, 128:418-424, 2001.

[7] M. Favino, C. Gross, M. Drolshagen, L. Keilig, C. Bourauel, J.Deschner, and R. Krause. Validation of a heterogeneous elastic-biphasic model for the numerical simulation of the PDL. Accepted for publication to Computer Methods in Biomechanics and Biomedical Engineering, 2011.

[8] V. Mow, W. Lai, and M. Holmes. Advanced theoretical and experimental techniques in cartilage research Biomechanics: principle and applications, 1, 1982.

[9] A. Quarteroni, R. Sacco, and F. Saleri. Numerical Mathematics. Springer, New York, 3rd edition, 2010. 